\title{
Radiographer reporting of trauma images: United Kingdom experience and the implications for evolving international practice
}

\author{
Maryann Hardy ${ }^{1}$, Beverly Snaith ${ }^{2}$, Tony Smith ${ }^{3}$ \\ 'University of Bradford, Bradford, United Kingdom. \\ ${ }^{2}$ Mid Yorkshire NHS Trust, United Kingdom. \\ ${ }^{3}$ Faculty of Health, The University of Newcastle, Newcastle 2300, Australia. \\ Correspondence: tony.smith@hnehealth.nsw.gov.au
}

\begin{abstract}
Some United Kingdom (UK) radiographers share the task of radiological reporting with radiologists. This paper aims to describe the current status of radiographer reporting of trauma images in the countries of the UK and reflect on its relevance to the development of similar advanced practice roles internationally.

In February 2007, a cross-sectional survey with respect to radiographer reporting was conducted of 456 UK hospitals with an Emergency Department (ED) or Minor Injuries Unit (MIU). The main outcome measures were the number of reporting radiographers; number of half-day radiographer reporting sessions per week; availability of, and radiographer involvement in 'hot' reporting. A total of $306(67.1 \%)$ responses were received. Reporting radiographers were employed at 56.9\% (174/306) of the respondent hospitals. Of hospitals with an ED, 70\% (142/203) employed reporting radiographers compared to $31.1 \%(32 / 103)$ of those with a MIU. At 146 of the 174 hospitals that employed reporting radiographers (83.9\%) there were between one and six radiographers involved. At 155 hospitals $(155 / 174 ; 89.1 \%)$ radiographers performed between 0 and 10 reporting sessions each week. Most reported for one or two sessions. 'Hot' reporting operated at only $11.1 \%$ (34/306) of the respondent hospitals, with radiographers leading this service at $64.7 \%$ (22/34) of those sites.

The rate of radiographer reporting uptake in UK hospitals appears to have slowed. Also, few radiographers are engaged in 'hot' reporting of trauma images. The prevalent UK model of 'cold' radiographer reporting may not be transferable to other countries where there is a need is for immediate and accurate reporting of trauma images.
\end{abstract}

Keywords: practice models, radiographer reporting, trauma radiography

\section{Introduction}

The reporting of trauma radiographs is an established extended role for United Kingdom (UK) radiographers. ${ }^{1}$ However, while the adoption of similar advanced practice roles is seen as desirable by radiographers internationally, acceptance and implementation of comparable initiatives has been slow. ${ }^{2,34}$ Radiographer reporting practice was introduced in the UK during the mid 1990s in response to growing service demand and increasing pressures on the radiology workforce. ${ }^{5}$ The role development has been underpinned and supported by postgraduate, higher education programs $^{6}$ and a credentialling process. Today, some radiographers now share the task of reporting radiographic images with radiologists in many UK hospitals ${ }^{7.8}$. Although the scope of individual radiographers' practice is usually limited to a single imaging modality (e.g. CT, MRI, ultrasound or conventional radiography), few limitations exist in terms of the referral pathways or in the range of anatomical regions and examination types covered. ${ }^{9}$

Initially, radiographer reporting was rapidly adopted into clinical practice. In 2004, a survey of radiographer role extension in the UK identified 94 hospital trusts (53\% of respondents) that employed radiographers to report musculoskeletal radiographs 9 . While this appeared to be a high proportion of hospitals, it equated to only 172 whole time equivalent (WTE) radiographers, or less than $1 \%$ of the radiography workforce in the UK at that time. ${ }^{10}$

It has been argued that the contribution of radiographers to the overall reporting workload has been limited by restrictions in their scope of practice and the availability of reporting time and space. ${ }^{11}$ This may be directly attributable to the service model of radiographer reporting adopted in the UK, which is based on the 'cold' or retrospective reporting traditionally provided by radiologists. However, with respect to trauma radiology services, the 'gold standard' is the availability of a report at the time of patient attendance, or 'hot' reporting. ${ }^{12}$

This paper reports the findings of a more recent survey of UK hospitals, the aims of which were to: assess the contribution of radiographers to the reporting of trauma radiographs; examine differences in radiographers' reporting role between countries in the UK; and gather background related to innovative practice models, those relating to 'hot' reporting in particular. It builds upon previously reported findings related to the role of the radiographer in trauma abnormality detection ${ }^{13}$ and explores some of the implications for the development of future practice models that may be internationally transferable.

\section{Method}

A cross-sectional survey was undertaken using a postal questionnaire, which was developed following a critical review of the literature. The questionnaire was pilot-tested on a small cohort of advanced practitioner and superintendent radiographers, who were asked to evaluate the appropriateness and relevance of the questions. The questionnaire was reviewed following feedback from the pilot study participants. 
Following institutional ethics committee approval in February 2007 , the survey was sent to all hospitals in the UK (including Northern Ireland, the Channel Islands and the Isle of Man) where there was either an Emergency Department (ED) or a Minor Injuries Unit (MIU), with medical imaging facilities. A total of 456 hospitals meeting these criteria were identified from the British Association of Emergency Medicine (BAEM) online directory. ${ }^{14}$ The questionnaire was mailed to the radiographer in charge of emergency imaging at each hospital.

The questionnaire sought data related to: the types of emergency care and medical imaging services provided; the hours of availability; and the extent to which radiographer abnormality detection schemes had been adopted. In addition, questions were asked about how the service operated and the contribution of radiographers to the trauma reporting workload.

Data analysis was undertaken using SPSS version 14.0 (SPSS Inc., Chicago, IL, USA) and STATA version 9.0 (Stata Corporation, College Station, TX, USA). Summary descriptive statistics of responses were generated, further detail of which is reported elsewhere. ${ }^{13}$ Comparison was also made between sites with an ED and those with a MIU.

\section{Results}

A total of $306(306 / 456=67.1 \%)$ responses were received. A greater proportion of responses came from hospitals that had an ED $(203 / 270=75.2 \%)$ as opposed to a MIU $(103 / 186$ in $55.4 \%)$ (z-test for proportional equivalence; $\mathrm{z}=1.99 ; P=0.047$ ).

Radiographers whose duties included reporting were employed at $56.9 \%(174 / 306)$ of the respondent hospitals (Table 1). A greater proportion of hospitals that operated an ED employed reporting radiographers $(142 / 203=70 . \%)$ compared to those that had a MIU $(32 / 103=31.1 \%)$. Variation in employment of reporting radiographers from country to country was also apparent. Of the countries from which 10 or more responses were received, England had the highest proportion of hospitals with reporting radiographers at just over $63 \%$ (Table 1 ).

Of the 174 hospitals that employed radiographers in a reporting role, only $146(146 / 174=83.9 \%)$ indicated the actual number of radiographers that performed reporting (Figure 1). This number ranged between 1 and 6, with a total of 363 radiographers distributed over these 146 sites (mean 2.5; median 2). Meanwhile, 155 hospitals $(155 / 174=89.1 \%)$ gave the number of half-day reporting sessions performed by radiographers (Figure 1), which ranged between 0 and 10 sessions per week $($ mean $=2.6$; median $=2$ ). Radiographers performed reporting for only one or two sessions

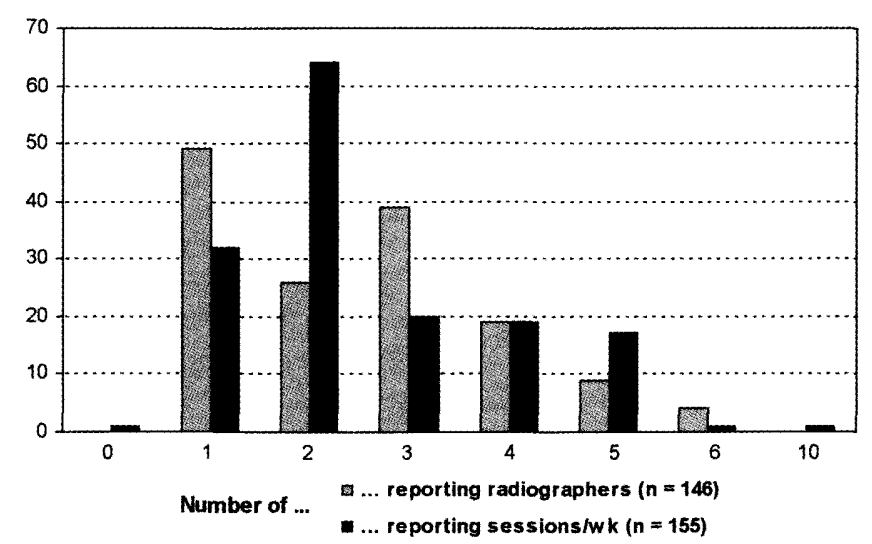

Fig. 1: Distributions of the number of reporting radiographers employed and number of radiographer reporting sessions per week at each hospital.

each week at $61.9 \%$ of these 155 hospitals.

A routine 'hot' reporting service for trauma patients operated at 34 of the hospitals that responded $(34 / 306=11.1 \%)$. This service was operated and led by radiographers at $64.7 \%(22 / 34)$ of these sites. A 'hot' reporting service was occasionally available at a further 23 hospitals $(23 / 306=7.5 \%)$.

\section{Discussion}

The accuracy of radiographers in correctly differentiating normal from abnormal appearances on conventional radiographic images has been examined extensively, including some large scale studies. ${ }^{15,16}$ Meta-analysis has demonstrated that, when compared to a 'reference standard', ${ }^{17}$ radiographers are capable of interpreting plain radiographs in clinical practice with a level of sensitivity of $92.6 \%$ (95\% CI: 92-93.2) and specificity of 97.7\% (95\% CI: 97.5-97.9). ${ }^{17}$ However, little published literature has considered radiographer reporting implementation strategies in the clinical setting, or the contribution of radiographer reporting to patient management, even though improvement in patient care is a primary objective of such skills mix initiatives..$^{18,19}$

It has been suggested that the number of radiographers contributing to the reporting workload in UK hospitals is increasing and that the trend is likely to continue. ${ }^{5.9}$ However, this survey suggests that the rate of increase in the utilisation of reporting radiographers is slowing compared to earlier survey results. ${ }^{9}$ In addition, with only about $38 \%$ of the sites surveyed providing radiographer reporting sessions for more than two half-days per week, there is evidence to suggest that demand for reporting radiographers may not be as great as was initially foreseen. However, contrary

Table 1: Employment of reporting radiographers and provision of a 'hot' reporting service by country $(n=306)$.

\begin{tabular}{lcccc}
\hline Country & Respondent hospitals & $\begin{array}{c}\text { Hospitals with reporting } \\
\text { radiographers }\end{array}$ & $\begin{array}{c}\text { Hospitals offering routine } \\
\text { 'hot' reporting }\end{array}$ & $\begin{array}{c}\text { Hospitals with } \\
\text { radiographer led routine } \\
\text { 'hot' reporting }\end{array}$ \\
\hline England & $236 / 347(68.0 \%)$ & $149 / 236(63.1 \%)$ & $29 / 236(12.3 \%)$ & $21 / 236(8.9 \%)$ \\
Scotland & $30 / 42(71.4 \%)$ & $5 / 30(16.7 \%)$ & $0 / 30$ & $0 / 30$ \\
Wales & $25 / 45(55.6 \%)$ & $13 / 25(52.0 \%)$ & $3 / 25(12.0 \%)$ & $1 / 25(4.0 \%)$ \\
Northern Ireland & $10 / 18(55.6 \%)$ & $5 / 10(50.0 \%)$ & $1 / 10(10.0 \%)$ & $0 / 10$ \\
Channel Islands & $3 / 3$ & $1 / 3(33.3 \%)$ & $0 / 3$ & $0 / 3$ \\
Isle of Man & $1 / 1$ & $0 / 1$ & $0 / 1$ & $0 / 1$ \\
Not stated & 1 & $1 / 1$ & $1 / 1$ & $0 / 1$ \\
\end{tabular}


arguments have suggested that radiographer reporting restrictions are a consequence of other factors, such as limited availability of reporting workstations and a shortfall of radiographers in the imaging workforce, which have reduced opportunities for both service and role development. ${ }^{11,20}$

The value of the radiographers' contribution to frontline image interpretation in the accident and emergency setting is well documented. ${ }^{3,12,17,18}$ It was the genesis of modern radiographer reporting, with the red dot system, ${ }^{21}$ and is the clinical area in which the potential benefit for patient outcomes is greatest. ${ }^{1}$ However, it appears that the proportion of UK hospitals providing routine 'hot' reporting of trauma examinations is low, at just over $11 \%$. Although radiographers lead the 'hot' reporting service in about $65 \%$ of hospitals where it operates, most reporting radiographers in the UK provide a retrospective ('cold') reporting service, in the traditional style of radiologists.

The formal, retrospective reporting of trauma radiographs serves a purpose, which is to identify abnormalities missed at the time of the patient's presentation, ${ }^{21}$ particularly in complex cases. However, the potential benefits to trauma services of radiographers providing an immediate, accurate report on trauma images appears to have been hastily overlooked in the push to develop this extended role. The substantial evidence that radiographer reporting accuracy can closely match that of radiologists in some areas has apparently encouraged hospitals to employ radiographers to fill gaps in their radiological reporting service. Perhaps, the need now is to modernise reporting services and to optimise the contribution of reporting radiographers to trauma care.

\section{International implications}

The international radiography community has watched the rapid evolution of radiographer reporting in the UK with interest, with a view to emulating this role development. It has been discussed in Australia, where some small scale studies have been conducted and some universities offer postgraduate image interpretation programs for radiographers. ${ }^{3}$ However, to date, little real progress has been made towards the development of a viable practice model.

Some radiographers in the United States have extended clinical roles related to performing and assisting with procedures. ${ }^{22}$ The responsibility for interpretation and reporting of images, however, remains with the radiologists. In South Africa and across the African continent, there has also been interest in the development of radiographer reporting from both within and outside the radiography profession, but as yet there has been limited success in implementing extended roles. ${ }^{4}$

It may be argued that the model of radiographer 'cold' reporting that is now the prevalent practice model in the UK is unlikely to be transferable in its entirety to other countries. Many of those countries have healthcare systems that are very different from the National Health Service (NHS). Australia, for example, has a much larger private radiology sector. The greatest benefit from developing an extended radiographer reporting role in Australia would be in frontline care of accident and emergency patients, particularly in the public healthcare system, and more so in rural and remote areas. The same is likely to be true in many other countries.

Those in the international medical imaging community who are in the process of planning or implementing a system of formal radiographer image interpretation and reporting may be well advised not to emulate the UK practice model, in which 'cold' reporting dominates. It may be better to explore innovative models that are relevant in the context of each country's healthcare system. In many cases, the need is for practice models that improve the immediacy and accuracy of 'hot' reporting of trauma radiographs.

Indeed, it may be timely and necessary to consider ways of better utilising the skills of reporting radiographers in the UK. The results of the survey reported above suggest varying degrees of underutilisation of the radiographer reporting capacity that has been created over the past decade. There is a need to ensure that skills are not eroded through limited opportunities to report on images in the clinical setting. Perhaps the time has come to consider the implementation of a practice model that encourages greater radiographer involvement in 'hot' reporting.

\section{Conclusions}

The results of the survey reported in this paper provide further insight into the contribution that radiographers make to reporting services, with particular reference to trauma imaging. The reporting role is now embedded in the professional field of diagnostic radiography in the $\mathrm{UK}$ and is continuing to evolve.

Evidence from this and previous surveys of radiographer role development indicates that more than half of UK hospitals employ radiographers to report on radiographic images. However, the initial rapid uptake of this extended role appears to be slowing and future changes in the proportion of health care facilities implementing radiographer reporting needs to be monitored to evaluate service trends. Further, an exploration of alternative practice models to those currently in place for the reporting of trauma images is recommended.

Despite 'hot' reporting of radiographic images at the time of the patient's presentation to the ED being regarded as the 'gold standard' for radiology service provision, relatively few UK hospitals with an ED or MIU employ radiographers in this role or indeed provide such a service. This appears contrary to the original intent of developing the radiographer reporting role. It is also argued above that the 'cold' reporting model commonly adopted across the UK may not be internationally transferable. Other countries require models that address the need for improvement in 'hot' reporting services. The UK is well placed to explore new models of frontline radiological diagnosis that may be more relevant to the international community than the 'cold' reporting model.

\section{References}

1 College of Radiographers. Medical image interpretation and clinical reporting by non-radiologists. London: College of Radiographers; 2005.

2 American Society of Radiologic Technologists. The Radiologist Assistant: Improving Patient Care While Providing Work Force Solutions. A consensus paper; 2002. Available online at: http://www.asrt.org/media/pdf/ra_consensuspaper.pdf [verified March 2008].

3 Smith TN, Baird M. Radiographers' role in radiological reporting: a model to support future demand. Med J Aust 2007; 186: 629-31.

4 Williams I. An international comparison - radiographer reporting in South Africa. Br J Radiol 2005; UK Radiology Congress Supplement.

5 Price R. Radiographer reporting: origins, demise and revival of plain film reporting. Radiography 2001; 7: 105-17.

6 Prime NJ, Paterson AM and Henderson PI. The development of a curriculum - a case study of six centres providing courses in radiographic reporting. Radiography 1999; 5: 63-70.

7 Benger JR, Lyburn ID. What is the effect of reporting all emergency department radiographs? Emerg Med J 2003; 20: 40-3.

8 Paterson AM, Price RC, Thomas A, Nuttall L. Reporting by radiographers: a policy and practice guide. Radiography 2004; 10: 205-10.

9 Price RC, Le Masurier SB. Longitudinal changes in extended roles in radiography: A new perspective. Radiography 2007; 13: 18-29.

10 Society of Radiographers. UK: Annual report. London: Society of Radiographers; 2004. 
11 Brayley N. The need for radiographer reporting: an accident and emergency (A\&E) perspective. Radiography 2000; 6: 227-9.

12 Audit Commission. Acute hospital portfolio: Radiology. London, UK: Audit Commission; 2002.

13 Snaith BA and Hardy ML. Radiographer abnormality detection schemes in the trauma environment - an assessment of current practice. Radiography (in press). Available online at www.sciencedirect.com/science/journal/10788174 [verified March 2008].

14 BAEM directory. London: British Association of Emergency Medicine; 2007. Available online at: http://www.baemdirectory.com [verified March 2008].

15 Robinson PJA, Culpan G, Wiggins M. Interpretation of selected accident and emergency radiographic examinations by radiographers: a review of 11000 cases. Br J Radiol 1999; 72: 546-51.

16 Piper KJ, Paterson AM, Godfrey RC. Accuracy of radiographers' reports in the interpretation of radiographic examinations of the skeletal system: A review of 6796 cases. Radiography 2005; 11: 27-34.

17 Brealey S, Scally A, Hahn S, Thomas N, Godfrey C, Coomarasamy A. Accuracy of radiographer plain film reporting in clinical practice: a metaanalysis. Clin Radiol 2005; 60: 232-41.
18 Royal College of Radiologists and Society and College of Radiographers. Team working within clinical imaging: A contemporary view of skills mix. London: Royal College of Radiologists; 2006.

19 Sibbald B, Shen J, McBride A. Changing the skill-mix of health care workforce. J Health Serv Res Policy 2004; 9: 28-38.

20 Royal College of Radiologists Workforce in crisis. London: Royal College of Radiologists; 2002.

21 Cheyne WN, Field-Boden QC, Wilson J, Hall R. The radiographer and the frontline diagnosis. Radiography 1987; 53: 114.

22 Guly HR. Diagnostic errors in an emergency department. Emerg Med J 2001; 18: 263-9.

23 Certification Board of Radiologist Practitioner Assistants (American Society of Radiologic Technologists, Albuquerque, NM); 2006. Available online at: www.cbrpa.org/pdf/RPApositiondescription.pdf [verified March 2008]. 